\section{Sandor Stephen Mester \\ Haym Benaroya}

Department of Mechanical and Aerospace Engineering

Rutgers, The State University of

New Jersey

New Brunswick, NJ

\title{
A Parameter Study of Localization
}

\begin{abstract}
Extensive work has been done on the vibration characteristics of perfectly periodic structures. Disorder in the periodic pattern has been found to lead to localization in one-dimensional periodic structures. It is important to understand localization because it causes energy to be concentrated near the disorder and may cause an overestimation of structural damping. A numerical study is conducted to obtain a better understanding of localization. It is found that any mode, even the first, can localize due to the presence of small imperfections. (C) 1996 John Wiley \& Sons, Inc.
\end{abstract}

\section{INTRODUCTION}

Several approaches have been used to analyze the dynamic behavior of periodic and near-periodic structures. The periodic nature of the structure is used to reduce the amount of computation required to find the solution. However, as a previous review by Mester and Benaroya (1995) substantiates, structures with slight deviations from a perfectly periodic pattern may have drastically different modal behavior than predicted by the periodic methods. Qualitatively, a localization criteria has been found to be the ratio between the amount of disorder and coupling strength. Although this criteria has been identified, work still needs to be done so that localization can be better understood, predicted quantitatively, and incorporated into the design process.

This work examined the difference between the mode shapes of periodic and near-periodic structures. The analysis illustrates the errors caused by not modeling small deviations when an idealized perfectly periodic model is assumed.
These errors only occur for certain structures possessing particular characteristics. The identification of these critical parameters governing this localization phenomenon has been continued. This investigation is based on a simulation of the one-dimensional structure in Fig. 1, consisting of 10 masses. Each mass represents the inertial properties of a substructure. Masses are attached to neighboring masses by a coupling spring, which represents the coupling stiffness between substructures. To represent the stiffness of a substructure, the mass is attached to a spring whose other end is fixed. The structure is assumed undamped.

\section{GENERAL METHOD OF SOLUTION}

The equation of motion for the structure shown in Fig. 1 is

$$
[M]\{\ddot{x}\}+[K]\{x\}=\{0\},
$$

Received January 8, 1995: Accepted August 1, 1995

Shock and Vibration, Vol. 3, No. 1, pp. 1-10 (1996)

(C) 1996 John Wiley \& Sons, Inc.

CCC $1070-9622 / 96 / 01001-10$ 


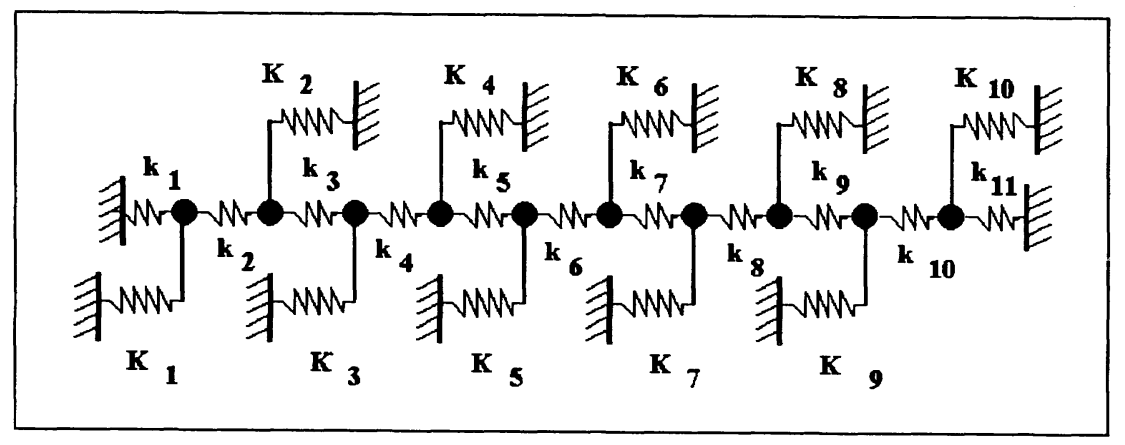

FIGURE 1 Baseline structure

where

$$
[M]=\left[\begin{array}{ccc}
m_{1} & \ldots & 0 \\
\vdots & \ddots & \vdots \\
0 & \ldots & m_{n}
\end{array}\right], \quad\{x\}=\left\{\begin{array}{c}
x_{1} \\
\vdots \\
x_{n}
\end{array}\right\},
$$

and

$$
[K]=\left[\begin{array}{cccc}
K_{1}^{\prime} & -k_{2} & \ldots & 0 \\
-k_{2} & \vdots & \vdots & \vdots \\
\vdots & \vdots & \vdots & -k_{n} \\
0 & \ldots & -k_{n} & K_{1}^{\prime}
\end{array}\right]
$$

The mass matrix, $[M]$, is a diagonal matrix and the stiffness matrix, $[K]$, is a tridiagonal matrix, where $K_{i}^{\prime}=k_{i}+K_{i}+k_{i+1}$. The number of degrees of freedom, $n$, is taken to be 10 in this study. To obtain the natural frequencies, $\Omega_{j}$, and modes shapes, $\left\{E V_{j}\right\}$, of the structure, the eigenvalue problem

$$
\left[\Omega^{2}[M]+[K]\right]\{E V\}=0
$$

needs to be solved by finding the values of $\Omega^{2}$ that satisfy

$$
\operatorname{det}\left|\Omega_{j}^{2}[M]+[K]\right|=0, \text { for } j=1 \cdots n \text {. }
$$

After the $\Omega_{j}$ 's have been determined, their corresponding mode shapes, $E V$, can be found from Eq. (2). The modes are ordered according to their frequencies, from the smallest frequency to the largest frequency.

The baseline structure in Fig. 1 is varied in different ways to observe how these changes affect the behavior of the mode shapes. Both a deterministic and stochastic model are considered. The deterministic model has a single prede- termined imperfection, while the stochastic model has a uniform random distribution of imperfections.

One parameter used to measure the deviation from an ideally periodic structure is the stiffness imperfection ratio (SIR). The $\mathrm{SIR}, \mathrm{SIR}=\Delta K /$ $K_{\text {nominal }}$, is the ratio of the greatest allowable change in a dynamic property to the nominal value of that dynamic property. A parameter that previously has been found to impact on the localization phenomena is the coupling stiffness ratio (CSR). This is the ratio of the nominal coupling stiffness between bays to the nominal bay stiffness, CSR $=k_{\mathrm{c}_{\text {nominal }}} / K_{\text {nominal }}$. A new parameter that is explored here is the disorder direction. The disorder direction simply indicates whether an imperfection increases or decreases the value of a dynamic property of a substructure. This study is confined to changes in stiffness. The disorder direction parameter is only applicable to the deterministic studies, because in the random analysis the stiffness values are randomly distributed about a nominal mean. However, the deterministic case gives insight to the random behavior, which will be discussed later.

Mode localization is the confinement of the near-periodic structure's mode shape to only a part of the structure in comparison with the mode shape of the perfectly periodic structure's mode shape. This is characterized by a mode shape that has sections that lie near the horizontal line $y=0$ for the imperfect structure but not for the perfectly periodic structure. Figure 2 gives an excellent illustration. The mode shape shown by the $\times$ 's is a highly localized mode. The mode shape drawn by the +'s is also localized, because the left half of the mode is nearly zero when compared with the mode shape of the idealized structure, represented by the $\mathrm{O}$ 's. Although both mode shapes are localized, it is easy 


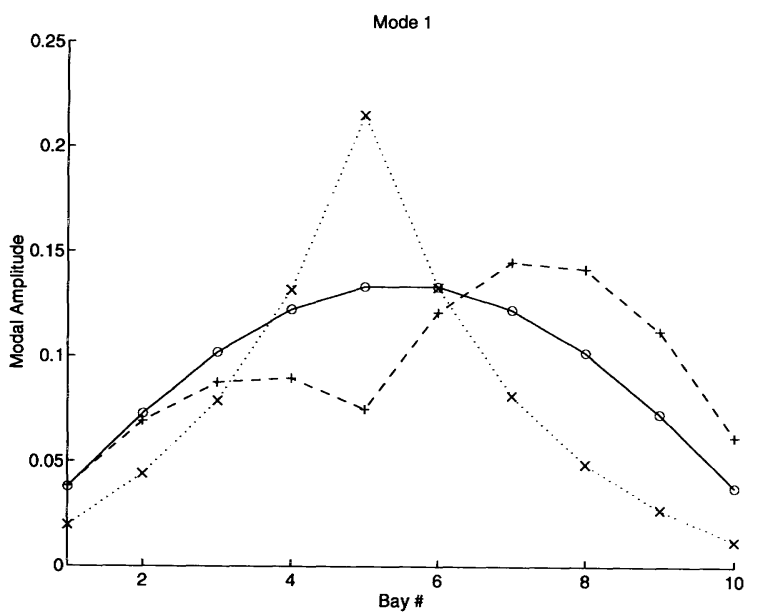

FIGURE 2 First mode shape with a disorder of $|\mathrm{SIR}|=10 \%$ at bay 5 and $\mathrm{CSR}=1 \% .(\times) \ominus$ disorder direction; $(\bigcirc)$ no imperfections; $(+) \oplus$ disorder direction.

to see that the localization is not to the same degree.

To quantify the degree of localization, a mathematical method needs to be introduced to measure this quantity. The degree of localization, $\sigma$, is computed as

$$
\sigma_{j}=\frac{\sum_{i=1}^{n}\left|\alpha_{i, j}\right|}{\sum_{i=1}^{n}\left|\beta_{i, j}\right|},
$$

where $\alpha_{i, j}$ is the $i$ th element of the $j$ th mode for the imperfect structure and $\beta_{i, j}$ is the corresponding element of the perfectly periodic structure. This method simply adds up the distance from $y=0$ to the points of the mode shape. This method generally works well, because a localized mode has many points that are very close to $y=$ 0 . Thus, lower $\sigma$ 's imply greater amounts of localization and higher $\sigma$ 's mean less modal localization. Returning to the example of Fig. 2, $\sigma$ for the mode shape drawn by the $\times$ 's is $40.7 \%$, and $\sigma$ is $79.6 \%$ for the other localized mode shape.

\section{DISORDER DIRECTION AND LOCATION ANALYSIS}

In this section, the effect of disorder direction and disorder location is examined. A disorder in a periodic structure is defined as a deviation from the nominal value of a structural property, i.e., stiffness, mass, or geometry. The disordered property is either greater than or less than the nominal value. The disorder direction is defined as positive $(+)$ when the disorder is greater than the nominal value, and negative $(-)$ when the disorder is less than the nominal value. Two "deviated" structures are modeled in Fig. 1 by either increasing or decreasing the cell stiffness of a single bay from the nominal stiffness by an equal amount. The resulting structures are then analyzed using the method described above. In the following figures, the points marked by,$+ \bigcirc$, and $\times$ represent mode shapes for a positive disorder direction $(+)$, no disorder $(O)$, and a negative disorder direction (-), respectively. For $\sigma$, the subscripts - and + indicate the degree of mode localization for a negative and a positive disorder direction, respectively.

\section{Disorder Direction}

To isolate the effect of disorder direction, the location of the disorder is restricted to the fifth mass while the parameters CSR and SIR are varied. Selected results are shown in Figs. 2-12. The mode shapes for both an increased and decreased stiffness, as well as the nominal stiffness, are depicted in Figs. 2-4 for SIR $=1,5$, and $10 \%$, respectively. The percent change of the modal amplitudes of each mass in these figures is given in Table 1.

In the case of very weak imperfection, SIR = $1 \%$, there is a slight localization of the mode $\left(\sigma_{-}=85 \%\right)$, as shown in Fig. 3. The modal amplitude of the fifth mass increases by $61 \%$ while

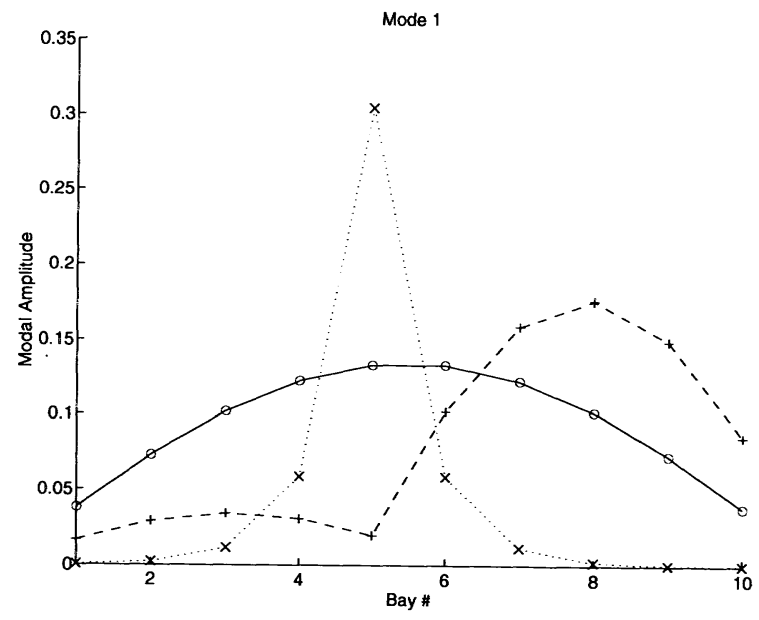

FIGURE 3 First mode shape with a disorder of $|\mathrm{SIR}|=1 \%$ at bay 5 and $\mathrm{CSR}=1 \% .(\times) \ominus$ disorder direction; $(\bigcirc)$ no imperfections; $(+) \oplus$ disorder direction. 
Table 1. Percent Change in First Modal Amplitudes from Baseline for CSR $=1 \%$

\begin{tabular}{|c|c|c|c|c|c|c|}
\hline \multirow[b]{3}{*}{ Mass } & \multicolumn{6}{|c|}{ Disorder Direction } \\
\hline & \multicolumn{2}{|c|}{$\operatorname{SIR}=1 \%$} & \multicolumn{2}{|c|}{$\mathrm{SIR}=5 \%$} & \multicolumn{2}{|c|}{$\operatorname{SIR}=10 \%$} \\
\hline & $\oplus$ & $\ominus$ & $\oplus$ & $\theta$ & $\oplus$ & $\theta$ \\
\hline 1 & 0.764 & -47.698 & -56.702 & -98.938 & -76.862 & -99.922 \\
\hline 2 & -4.74 & -39.199 & -60.507 & -97.019 & -79.01 & -99.583 \\
\hline 3 & -13.935 & -22.469 & -66.689 & -88.911 & -82.485 & -96.987 \\
\hline 4 & -26.896 & 7.561 & -75.031 & -52.157 & -87.142 & -74.72 \\
\hline 5 & -43.834 & 61.312 & -85.27 & 128.302 & -92.803 & 134.62 \\
\hline 6 & -9.122 & -0.304 & -23.513 & -56.033 & -27.32 & -76.768 \\
\hline 7 & 18.285 & -33.524 & 29.652 & -90.786 & 29.849 & -97.497 \\
\hline 8 & 39.256 & -52.083 & 72.968 & -97.864 & 76.886 & -99.702 \\
\hline 9 & 54.134 & -62.423 & 105.073 & -99.426 & 111.983 & -99.959 \\
\hline 10 & 63.04 & -67.676 & 124.831 & -99.795 & 133.673 & -99.992 \\
\hline
\end{tabular}

the modal amplitudes of the other masses decrease for the case of decreased stiffness (negative disorder direction). The mode corresponding to increased stiffness does not become localized $\left(\sigma_{+}=101 \%\right)$, but the mode shape changes such that the modal amplitudes of the masses to the left of the imperfection diminish slightly while those of the masses to the left increase slightly.

By increasing the imperfection, SIR $=5 \%$ (Fig. 4) the localization becomes more pronounced. The modal amplitude of the fifth mass increases by $128 \%$, while the rest of the modal amplitudes are negligible for the case of $(-)$ disorder direction $\left(\sigma_{-}=48 \%\right)$. For the case of $(+)$ disorder direction, the modal amplitudes of the masses to the left of the fifth mass become negli-

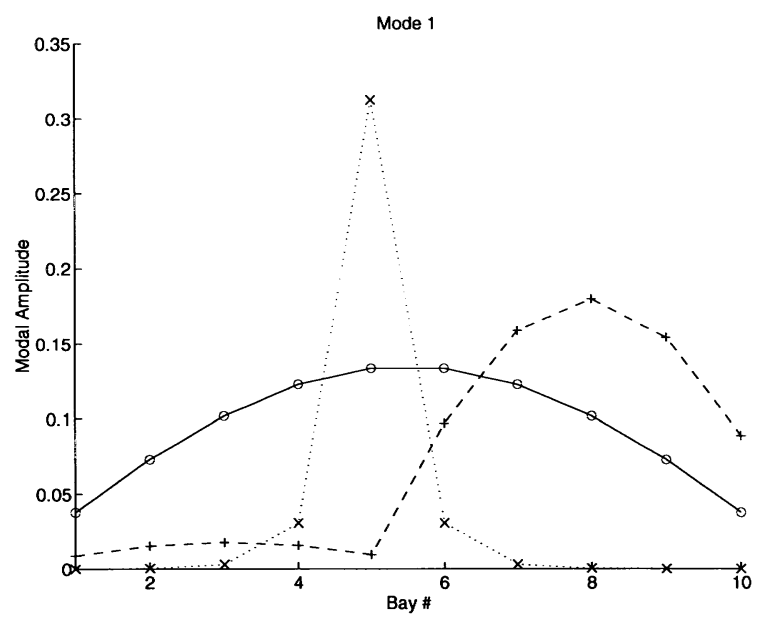

FIGURE 4 First mode shape with a disorder of $|\mathrm{SIR}|=5 \%$ at bay 5 and $\mathrm{CSR}=1 \% .(\times) \ominus$ disorder direction; $(\bigcirc)$ no imperfections; $(+) \oplus$ disorder direction. gible (-99.8\%) with respect to the masses on the right of the fifth mass. In essence, the mode becomes localized to the right part of the system $\left(\sigma_{+}=85 \%\right)$.

An increase to an imperfection of SIR $=10 \%$ (Fig. 2) causes little change from the results given by SIR $=5 \%$. The mode shapes for the two cases are the same, although the case of greater imperfection has slightly greater extremes $\left(\sigma_{+}=\right.$ $80 \%, \sigma_{-}=41 \%$ ). That is, the masses that had increased modal amplitudes in the case of SIR = $5 \%$ have slightly greater modal amplitudes. The modal amplitudes that were negligible remain negligible. These results for the negative disorder direction agree with results found by other investigators, e.g., Cha and Pierre (1991): as the

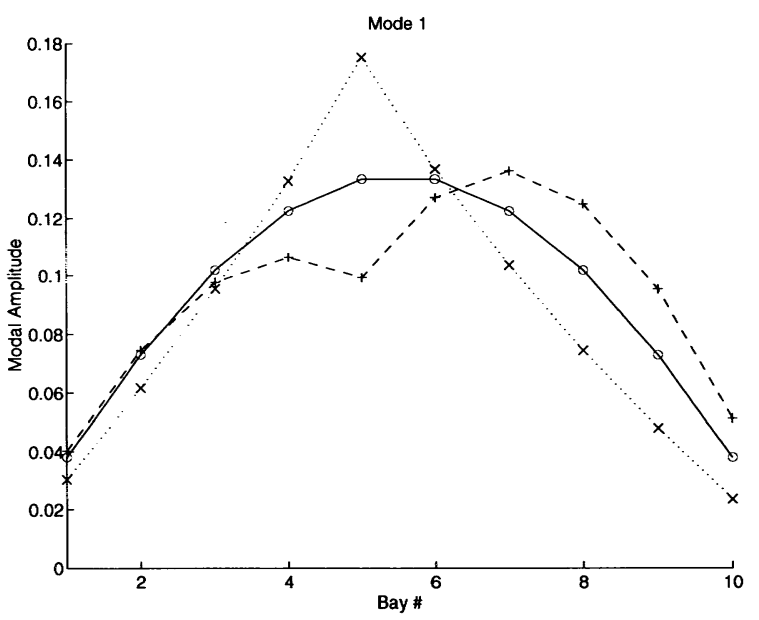

FIGURE 5 First mode shape with a disorder of $|\mathrm{SIR}|=5 \%$ at bay 5 and $\mathrm{CSR}=10 \% .(\times) \ominus$ disorder direction; $(O)$ no imperfections; $(+) \oplus$ disorder direction. 

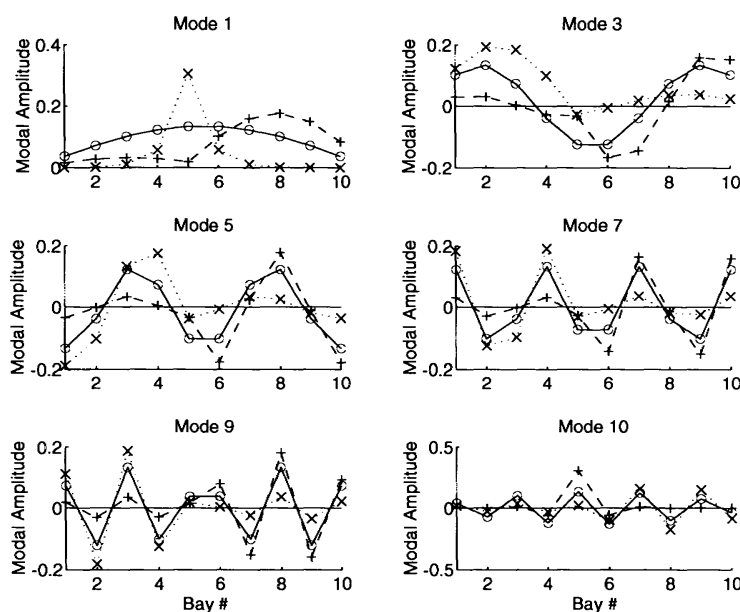

FIGURE 6 Selected modes for $\mid$ SIR $\mid=5 \%$ at bay 5 and $\mathrm{CSR}=1 \% .(\times) \ominus$ disorder direction: $(\bigcirc)$ no imperfections; $(+) \oplus$ disorder direction.

strength of the imperfection increases, the localization of modes increases.

\section{Coupling Strength}

The effect of coupling strength is shown in Figs. 4 and 5 where the SIR $=5 \%$. In Fig. 4, the coupling strength is weak, CSR $=1 \%$, and the modes localize due to the imperfection in the structure. However, by increasing the coupling strength by an order of magnitude to CSR $=10 \%$ (Fig. 5) the modes of the disordered structures approach the mode shape of the perfect structure. This also agrees with previously observed behavior (Hodges, 1982) that as the coupling strength increases, mode localization decreases.

A noteworthy observation from this study is found by looking at Figs. 6 and 7. The first mode in both figures localizes to a greater extent for a negative disorder direction $\left(\sigma_{-}=48\right.$ and $\left.41 \%\right)$, than for the positive disorder direction $\left(\sigma_{+}=85\right.$ and $80 \%$ ). The last mode (10th) behaves in a converse manner. The last mode localizes more due to a positive disorder direction $\left(\sigma_{+}=48\right.$ and $41 \%$ ) than when the disorder direction is negative $\left(\sigma_{-}=85\right.$ and $\left.80 \%\right)$. A similar effect can be seen on the natural frequencies (refer to Table 2). The first mode has a shift of -1.7 and $-4.23 \%$ in its natural frequency due to a negative disorder direction for SIR $=5$ and $10 \%$, respectively. The 10 th mode's natural frequency is shifted, 1.7 and $3.9 \%$ by a positive disorder direction. These shifts are significant when compared to the rest of the modes that have natural frequency shifts
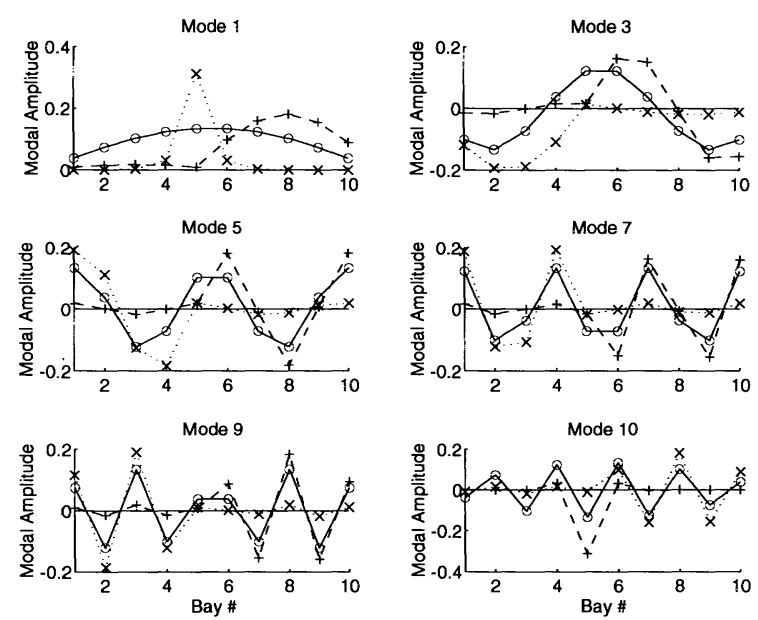

FIGURE 7 Selected modes for $|\operatorname{SIR}|=5 \%$ at bay 5 and $\operatorname{CSR}=10 \%$. $(\times) \ominus$ disorder direction; $(\bigcirc)$ no imperfections; $(+) \oplus$ disorder direction.

of less than $0.15 \%$. These observations are generalized as follows. A decrease in stiffness has a greater effect on the lower modes of the structure: the lower the mode, the greater the effect. The converse is also true. That is, an increase in stiffness causes a more pronounced change, the higher the mode. Thus it follows that the modes in the center are not greatly affected by a change in stiffness. Also, the change tends to be approximately the same whether the stiffness is increased or decreased for these central modes.

\section{Disorder Location}

The mode shape generally localizes at the location of the disorder. Mode localization is when portions of the mode, which are not originally zero for the idealized structure, become nearly equal to zero by the inclusion of an imperfection in the analysis. Naturally, the nonzero portion of

Table 2 Percent Change in Natural Frequency from Baseline, CSR $=1 \%$

\begin{tabular}{clcccc}
\hline & \multicolumn{4}{c}{ Disorder Direction } \\
\cline { 2 - 3 } & \multicolumn{2}{c}{ SIR $=5 \%$} & & \multicolumn{2}{c}{ SIR $=10 \%$} \\
\cline { 2 - 3 } \cline { 5 - 6 } Mode & $\oplus$ & & & $\oplus$ & $\ominus$ \\
\hline 1 & 0.08 & -1.7 & & 0.09 & -4.23 \\
3 & 0.13 & -0.13 & & 0.15 & -0.14 \\
5 & 0.11 & -0.12 & & 0.13 & -0.14 \\
7 & 0.08 & -0.08 & & 0.07 & -0.09 \\
10 & 1.7 & -0.08 & & 3.9 & -0.09 \\
\hline
\end{tabular}




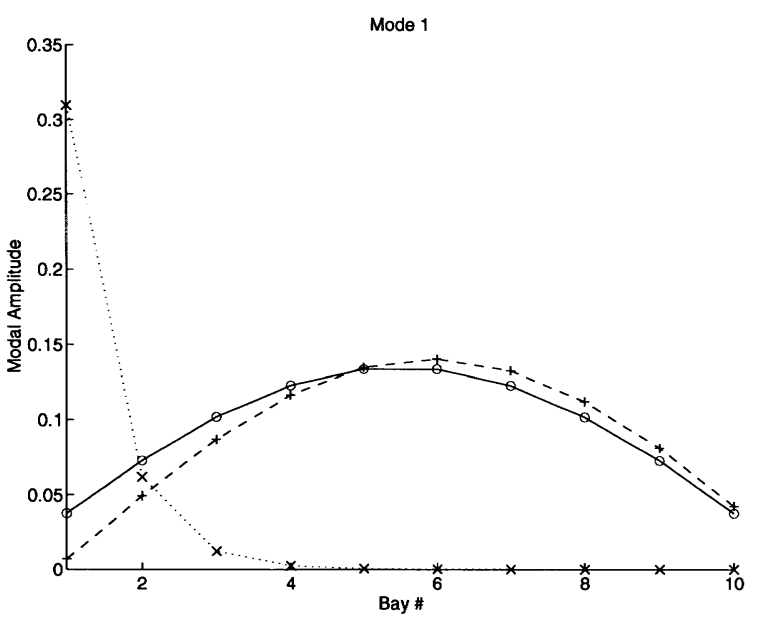

FIGURE 8 First mode shape with a disorder of $|\mathrm{SIR}|=5 \%$ at bay 1 and $\operatorname{CSR}=1 \% .(\times) \ominus$ disorder direction; $(\bigcirc)$ no imperfections; $(+) \oplus$ disorder direction.

the mode is the region considered to be localized. In other words, the modal energy becomes confined to that area. A mathematical description of the localized region is given as follows:

$$
\frac{\left|\alpha_{i, j}\right|}{\left|\beta_{i, j}\right|} \geq 1 \text {. }
$$

So, the $j$ th mode of the disordered system is localized in the bays where the modal amplitude is equal to or larger than the corresponding modal amplitude of the idealized structure. This can be

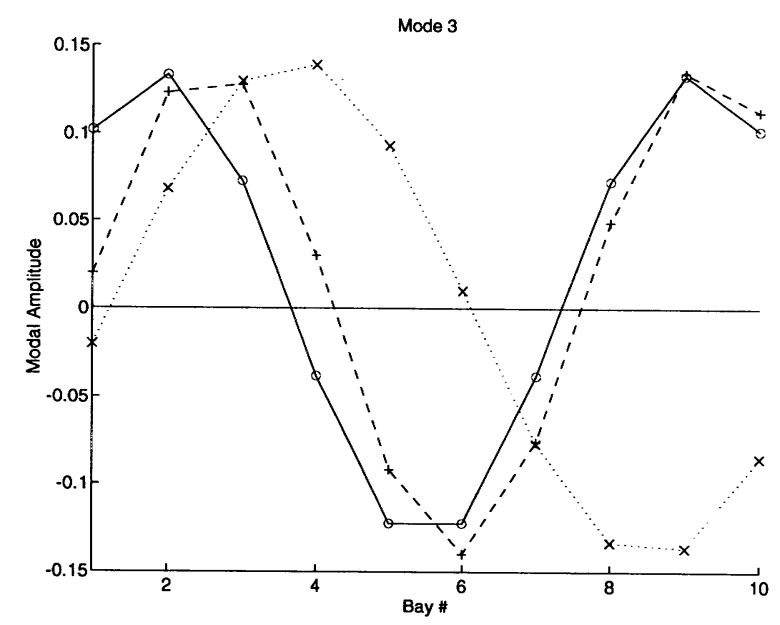

FIGURE 9 Third mode shape with a disorder of $|\mathrm{SIR}|=5 \%$ at bay 1 and $\mathrm{CSR}=1 \% .(\times) \ominus$ disorder direction; $(\bigcirc)$ no imperfections; $(+) \oplus$ disorder direction.

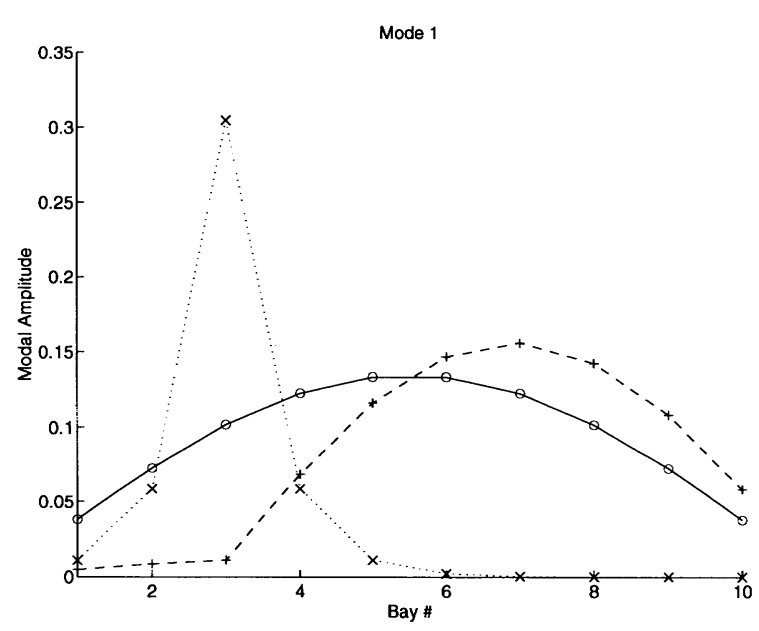

FIGURE 10 First mode shape with a disorder of $|\mathrm{SIR}|=5 \%$ at bay 3 and $\mathrm{CSR}=1 \% .(\times) \ominus$ disorder direction; $(\bigcirc)$ no imperfections; $(+) \oplus$ disorder direction.

seen in Figs. 2 and $8-12$. In the case where the disorder is located in the first bay (Fig. 8) the first mode shape localizes for the case of decreased stiffness $\left(\sigma_{-}=41 \%\right)$, but increasing the stiffness has virtually no effect $\left(\sigma_{+}=96 \%\right)$. The same holds true for the third mode shape with respect to increasing the stiffness $\left(\sigma_{+}=97 \%\right.$, Fig. 9). However, decreasing the stiffness does not localize the mode shape $\left(\sigma_{-}=95 \%\right)$, but the mode shape is altered such that it appears to be more like the perfect second mode shape (refer to Fig. 13).

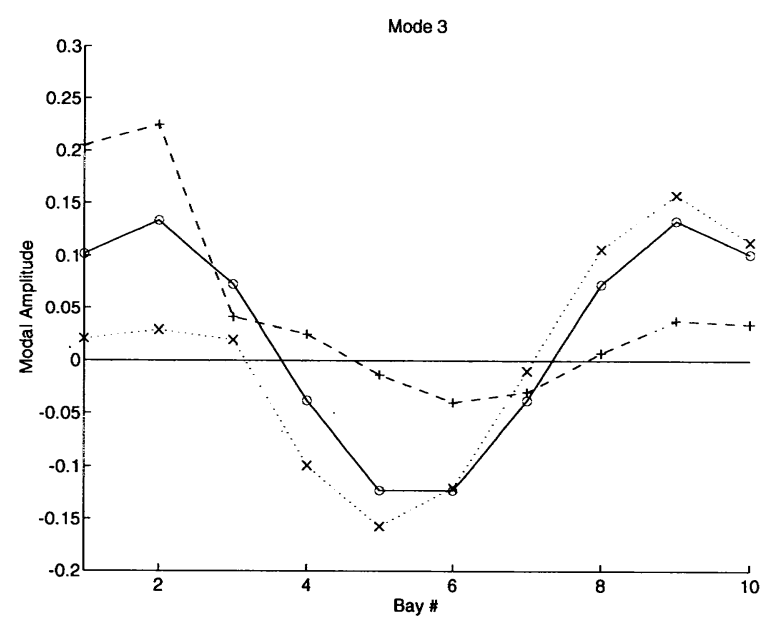

FIGURE 11 Third mode shape with a disorder of $|\mathrm{SIR}|=5 \%$ at bay 3 and $\mathrm{CSR}=1 \% .(\times) \ominus$ disorder direction; $(\bigcirc)$ no imperfections; $(+) \oplus$ disorder direction. 
For the case where the disorder is located at bay 3 (Fig. 10), similar results are obtained. Again, the first mode shape is localized at bay 3 for decreased stiffness $\left(\sigma_{-}=48 \%\right)$. The effect on the first mode shape of increasing the cell stiffness is that the modal amplitude of bays 1,2, and 3 are insignificant compared to the rest of the structure, confining most of the modal energy to the right of bay $3\left(\sigma_{+}=88 \%\right)$. The third mode shape for a disorder located at the third bay (Fig. 11) localizes to the left of bay 3 for increased stiffness $\left(\sigma_{+}=70 \%\right)$ and to the right of bay 3 for decreased stiffness $\left(\sigma_{-}=88 \%\right)$.

Finally, for an imperfection with a negative disorder direction located at the fifth bay, the first mode shape (Fig. 2) localizes at the disorder, while most of the response is to the right of the fifth bay when the disorder direction is reversed. Also, the third mode shape for a disorder at the fifth bay (Fig. 12) behaves similarly to the case above when the disorder is located at the third bay. That is, the mode localizes to one side due to a positive disorder and to the other side for a negative disorder.

It is interesting to note that the modal amplitude of the mode increases as the number of bays over which the energy is confined decreases. For example, comparing Figs. 11 and 12, the maximum modal amplitude of the third mode shape, for the increased stiffness case, is approximately $50 \%$ greater when the disorder is at the third bay as when the disorder is at the fifth bay. This seems intuitive due to the conservation of en-

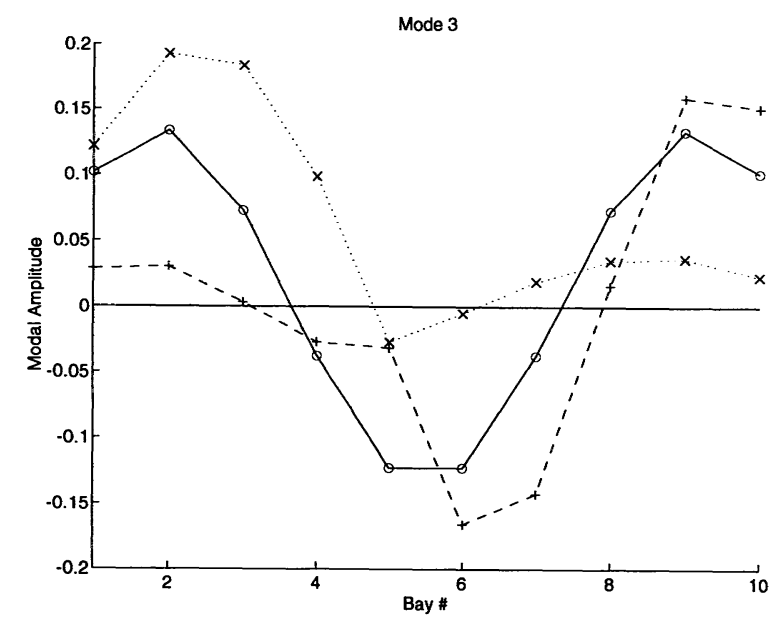

FIGURE 12 Third mode shape with a disorder of $|\mathrm{SIR}|=5 \%$ at bay 5 and $\mathrm{CSR}=1 \% .(\times) \ominus$ disorder direction; $(O)$ no imperfections; $(+) \oplus$ disorder direction.

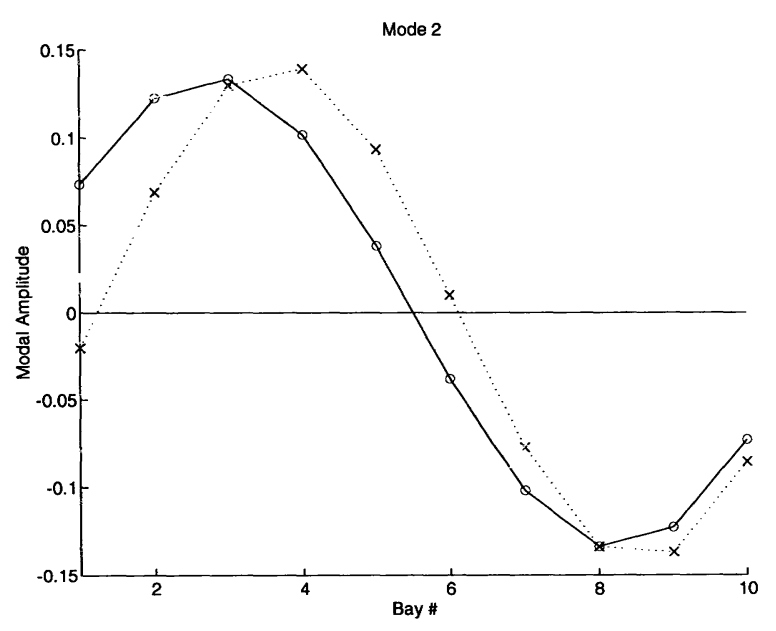

FIGURE 13 Mode 2 of perfect structure vs. mode 3 of disordered structure with $\mathrm{CSR}=1 \%, \mathrm{SIR}=-5 \%$ at bay 1. $(\times) \ominus$ disorder direction; $(O)$ no imperfections.

ergy. The energy in the first case is confined over three bays, while in the second case it is confined over six bays.

\section{RANDOM DISTRIBUTION OF STIFFNESS PROPERTIES}

This analysis is a generalization of the previous single disorder studies. In this case, the masses of the baseline structure (Fig. 1) are attached to springs that have randomly distributed stiffness properties. The probability distribution for the stiffness is modeled as uniform, which is a rea-

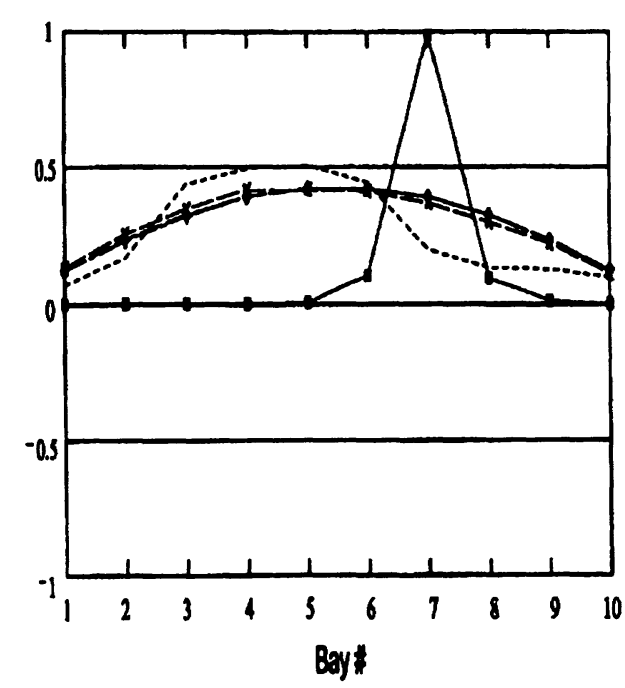

FIGURE 14 Mode 1 random stiffness SIR $=1 \%$. 


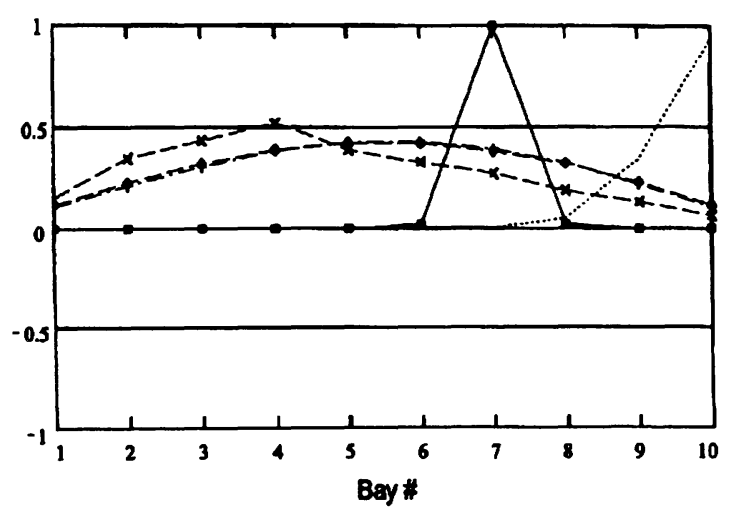

FIGURE 15 Mode 1 random stiffness SIR $=5 \%$.

sonable choice where only a range is known for the parameter.

Some results are shown in Figs. 14-16. In each of the three figures, the first mode shape is plotted. The natural frequencies of each bay of the structures depicted in Figs. 14-16 are given in Tables 3-5, respectively. Four orders of magnitude are considered for the coupling stiffness ratio ranging from 0.1 to $1000 \%$ in each of the figures. In Fig. 14, the stiffnesses of the springs vary uniformly $( \pm 1 \%)$ about their nominal value, SIR $=1 \%$. The nominal value of the coupling springs, $k_{c_{\text {num }}}$, is 1 . The nominal value of the cell stiffness is determined by the coupling stiffness ratio $\left(K_{\text {nom }}=k_{c_{\text {nom }}} / \mathrm{CSR}\right)$. The modes only become localized for the two weakest coupling cases of CSR $=0.001$ and 0.01 .

As the strength of the disorder increases (Fig. 15) to SIR $=5 \%$, the localization of the mode for $\mathrm{CSR}=0.01$ becomes more pronounced. Also, the mode for CSR $=0.1$ becomes localized. An observation, seen in Fig. 15, is that the mode tends to localize at the bays that have the lowest natural frequencies. For the mode associated with CSR $=0.001$, the mode localizes in bay 7 ,

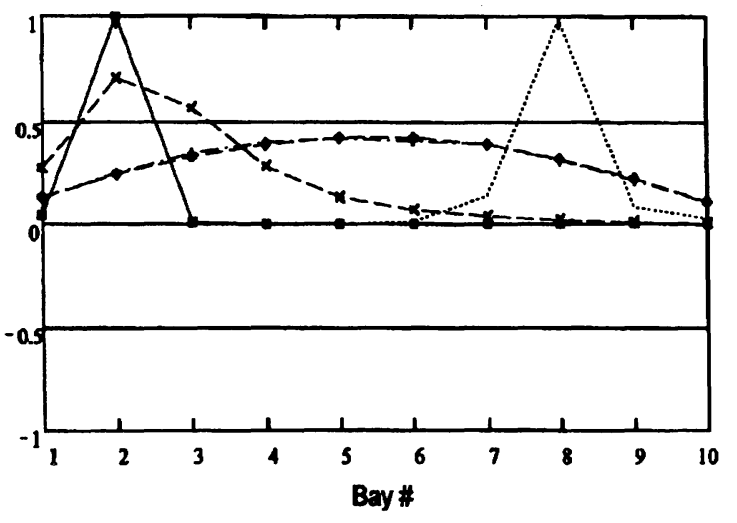

FIGURE 16 Mode 1 random stiffness SIR $=10 \%$.

which has a natural frequency of 30.87. Similarly, the mode for CSR $=0.01$ localizes at bay 10 , which has a natural frequency of 9.75 . Mode 1 for the structure with CSR $=0.1$ is partially localized about bay 4 . The natural frequency of bay 4 is 3.10 . The next lowest natural frequency is 3.12 in bay 2 .

Finally, at SIR $=10 \%$, the first mode localizes for the three structures that have CSR of 0.001 , 0.01 , and 0.1 (Fig. 16). Again the modes localize in the bays with the lowest natural frequencies. For CSR $=0.001$, the frequency of the localized bay is 30.03 , which is the lowest of all the bays. The lowest bay natural frequency of the structure with CSR $=0.01$ is 9.76 in bay 8 . The localization of the first mode of the structure with CSR $=0.1$ is located in the second and third bay, which are the two lowest natural bay frequencies.

This behavior is similar to that exhibited by the disorder direction analysis in the preceding section. In the deterministic case, the mode localizes at the bay where the disorder occurs for a negative disorder direction. The negative disorder effectively lowers the natural frequency of the disordered bay relative to the rest of the

Table 3 Natural Frequencies of Bays of Structure with Uniform Random Distribution of SIR $=1 \%$

\begin{tabular}{lrrrrrrrrrrr}
\hline & \multicolumn{10}{c}{ Bay No. } \\
\cline { 2 - 13 } CSR & \multicolumn{1}{c}{1} & \multicolumn{1}{c}{ 1 } & \multicolumn{1}{c}{3} & \multicolumn{1}{c}{4} & \multicolumn{1}{c}{5} & \multicolumn{1}{c}{6} & \multicolumn{1}{c}{7} & \multicolumn{1}{c}{8} & \multicolumn{1}{c}{9} & 10 & $\Omega$ \\
\hline 0.001 & 31.61 & 31.78 & 31.70 & 31.53 & 31.73 & 31.62 & 31.47 & 31.65 & 31.63 & 31.73 & 31.50 \\
0.01 & 10.02 & 10.03 & 9.96 & 9.98 & 9.98 & 9.96 & 10.03 & 10.01 & 9.98 & 9.95 & 9.99 \\
0.1 & 3.17 & 3.15 & 3.16 & 3.15 & 3.17 & 3.16 & 3.16 & 3.17 & 3.16 & 3.16 & 3.17 \\
1 & 1.00 & 1.00 & 1.00 & 1.00 & 1.00 & 1.00 & 1.00 & 1.00 & 1.00 & 1.00 & 1.04 \\
10 & 0.32 & 0.32 & 0.32 & 0.32 & 0.32 & 0.32 & 0.32 & 0.32 & 0.32 & 0.32 & 0.43 \\
\hline
\end{tabular}


Table 4 Natural Frequencies of Bays of Structure with Uniform Random Distribution of SIR = 5\%

\begin{tabular}{|c|c|c|c|c|c|c|c|c|c|c|c|}
\hline \multirow[b]{2}{*}{ CSR } & \multicolumn{11}{|c|}{ Bay No. } \\
\hline & 1 & 2 & 3 & 4 & 5 & 6 & 7 & 8 & 9 & 10 & $\Omega$ \\
\hline 0.001 & 31.55 & 32.38 & 32.00 & 31.14 & 32.15 & 31.62 & 30.87 & 31.74 & 31.67 & 32.16 & 30.90 \\
\hline 0.01 & 10.08 & 10.17 & 9.80 & 9.91 & 9.89 & 9.82 & 10.17 & 10.05 & 9.88 & 9.75 & 9.83 \\
\hline 0.1 & 3.21 & 3.12 & 3.17 & 3.10 & 3.20 & 3.17 & 3.15 & 3.19 & 3.15 & 3.17 & 3.17 \\
\hline 1 & 1.01 & 1.00 & 1.01 & 0.98 & 0.98 & 1.00 & 0.98 & 0.98 & 1.02 & 1.02 & 1.03 \\
\hline 10 & 0.31 & 0.31 & 0.32 & 0.32 & 0.32 & 0.32 & 0.32 & 0.32 & 0.31 & 0.32 & 0.43 \\
\hline
\end{tabular}

structure. Hence, slight deviations in the natural frequencies of the bays significantly change the mode shapes of the structure. It seems that the location of the modal localization may be determined by finding the bay with the lowest natural frequency.

\section{SUMMARY}

Methods such as transfer matrices, wave propagation, and power propagation for the analysis of periodic and near-periodic structures have been explored in the literature. These studies have shown that small imperfections in weakly coupled structures cause localization of energy about the imperfection.

This study shows that slight deviations from a perfectly ordered structure cause substantial changes in the structural mode shapes. A mathematical definition for mode localization was introduced. Also, the region of modal confinement was defined. It was also demonstrated that the localization phenomenon is not limited to long structures. Even the first mode of a short structure can localize from the presence of most ("engineering") imperfections. As a result, the actual response of a structure may be quite different than that predicted by a periodic analysis method. Thus, in designing and/or controlling a structure, it is important to account for imperfections. Also, it has been shown that the high and low modes are more susceptible to imperfections than the central modes. An important finding is that the sensitivity to imperfections of the end modes is disorder direction dependent. The deterministic study shows that the disorder location has a direct effect on mode localization, such that the mode localizes either at the disorder or to one side of the disorder, depending on the disorder direction. The trends shown in this study give a better understanding of mode localization.

The random disorder distribution is found to be the generalization of the deterministic study. Again, the first and last modes are more sensitive to imperfection, as it is in the deterministic case. The mode localization seems to occur in the bays that have extreme natural frequencies. This may prove to be useful in nondestructive testing and locating imperfections that develop during the life of near-periodic structures.

The next step in this study is to determine the overall response of a near-periodic structure to determine how the localized modes influence the response. Also, efforts to develop possible control strategies in which this type of behavior is advantageous will be pursued.

Table 5 Natural Frequencies of Bays of Structure with Uniform Random Distribution of SIR $=10 \%$

\begin{tabular}{|c|c|c|c|c|c|c|c|c|c|c|c|}
\hline \multirow[b]{2}{*}{ CSR } & \multicolumn{11}{|c|}{ Bay No. } \\
\hline & 1 & 2 & 3 & 4 & 5 & 6 & 7 & 8 & 9 & 10 & $\Omega$ \\
\hline 0.001 & 30.39 & 30.03 & 31.72 & 31.94 & 30.55 & 31.47 & 30.19 & 32.51 & 31.69 & 32.79 & 30.06 \\
\hline 0.01 & 10.45 & 10.04 & 9.96 & 10.36 & 10.28 & 10.49 & 10.11 & 9.76 & 10.33 & 9.88 & 9.85 \\
\hline 0.1 & 3.22 & 3.00 & 3.09 & 3.19 & 3.27 & 3.16 & 3.24 & 3.15 & 3.24 & 3.19 & 3.13 \\
\hline 1 & 1.02 & 1.01 & 0.96 & 0.99 & 1.00 & 1.02 & 0.97 & 1.00 & 1.02 & 0.96 & 1.03 \\
\hline 10 & 0.30 & 0.32 & 0.31 & 0.33 & 0.31 & 0.33 & 0.31 & 0.33 & 0.31 & 0.32 & 0.42 \\
\hline
\end{tabular}


This work was prepared in partial fulfillment of the requirements for the degree of Doctor of Philosophy of the first author. The Federal Aviation Administration Technical Center is the supporter of this work.

\section{REFERENCES}

Cha, P. D., and Pierre, C., 1991, "Vibration localization by disorder in assemblies of monocoupled, multimode component systems," Journal of Applied Mechanics, Vol. 58, pp. 1-10.

Hodges, C. H., 1982, "Confinement of vibration by structural irregularity," Journal of Sound and Vibration, Vol. 82, pp. 411-424.

Mester, S., and Benaroya, H., 1995, "A review of periodic and near periodic structures," Shock and Vibration, Vol. 2, pp. 69-95. 

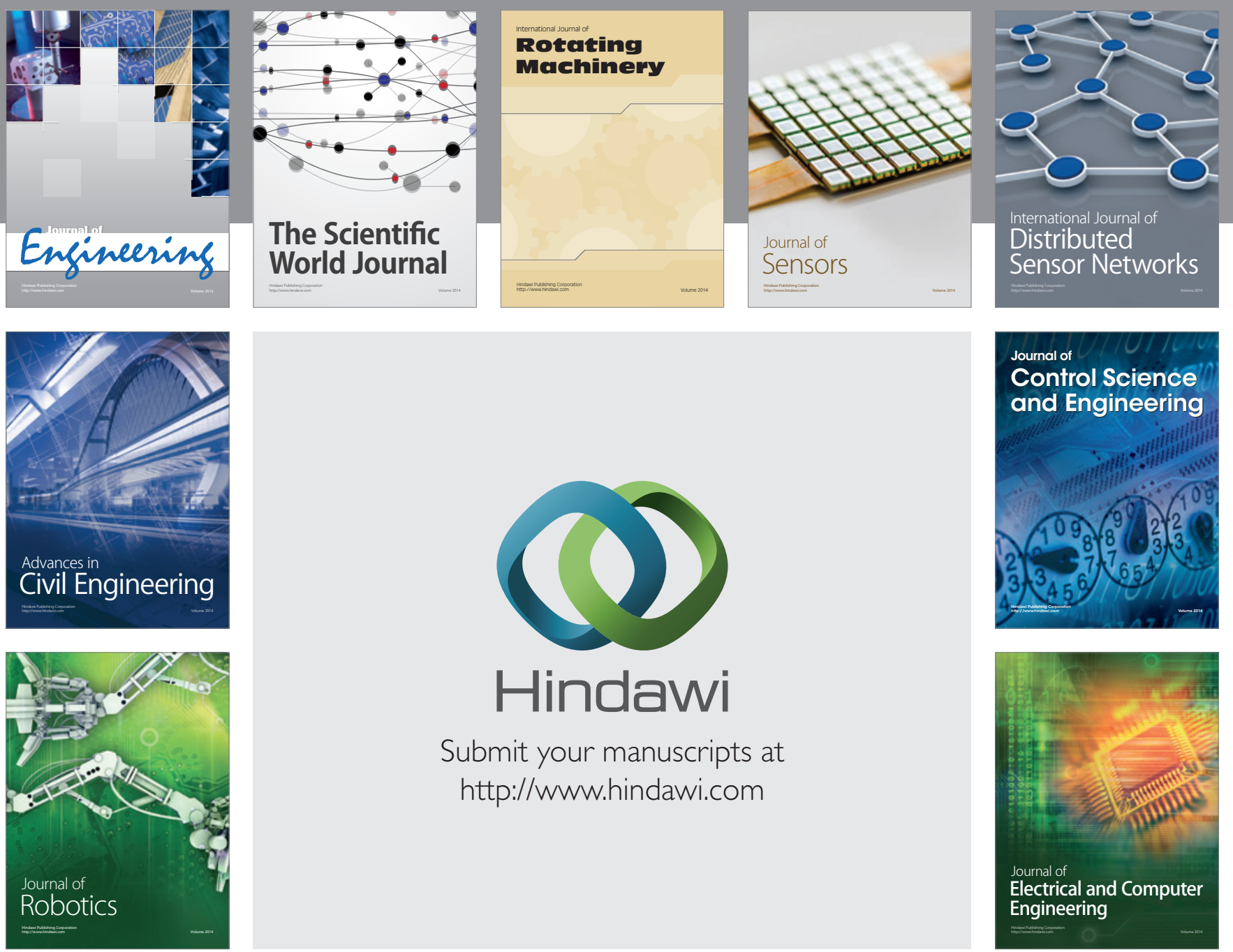

Submit your manuscripts at

http://www.hindawi.com
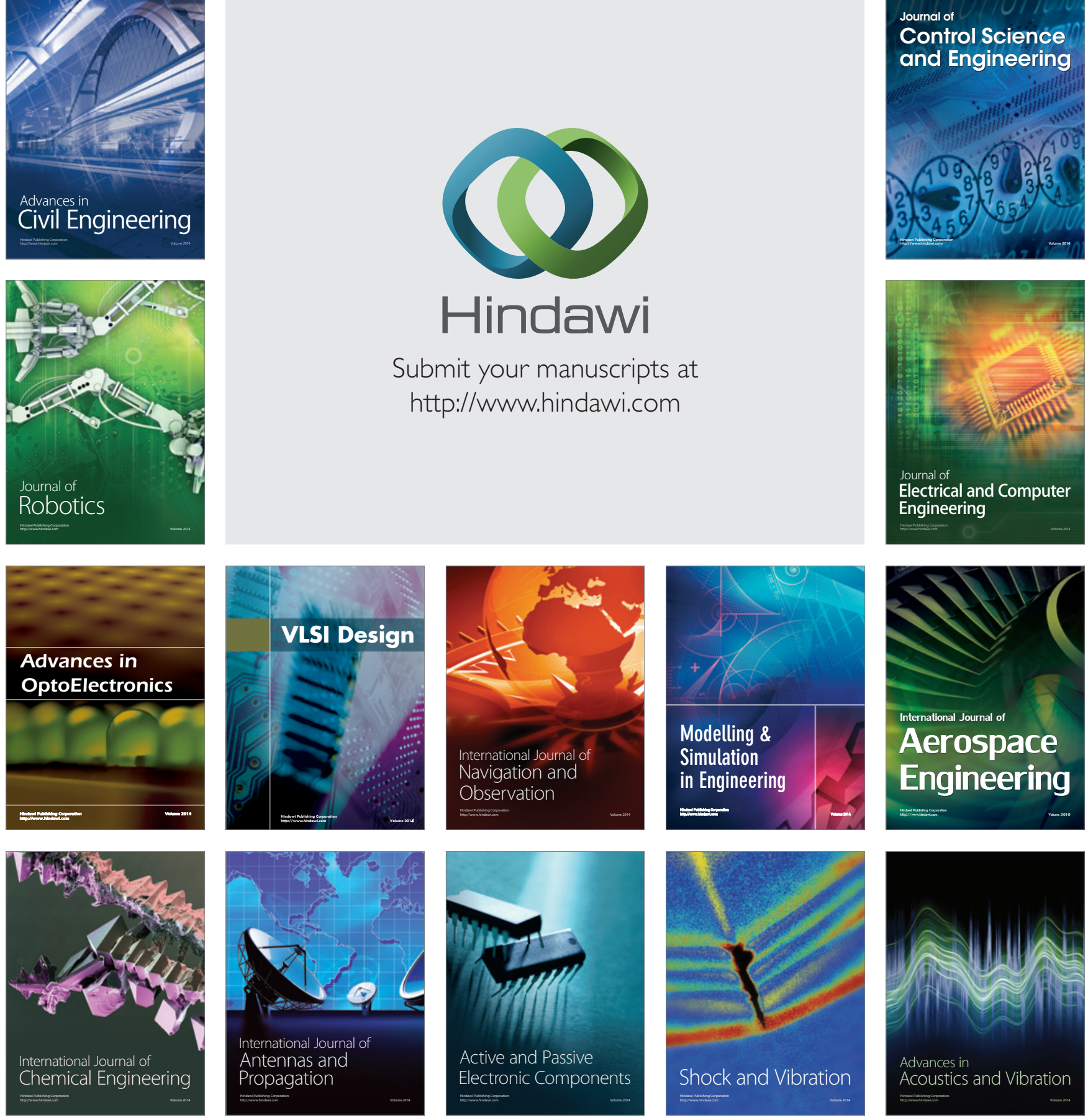